\title{
ESTRATEGIAS DE MERCADOTECNIA EN \\ MICROEMPRESAS ANTE LA CONTINGENCIA POR \\ COVID-19 EN CIUDAD JUÁREZ, MÉXICO: UN ESTUDIO \\ CUALITATIVO
}

\author{
MARKETING STRATEGIES IN MICRO-ENTERPRISES IN THE FACE OF THE \\ COVID-19 CONTINGENCY IN CIUDAD JUÁREZ, MEXICO: A QUALITATIVE STUDY
}

\author{
Fany Thelma Solís-Rodríguez ${ }^{1 *}$ (D) . \\ 1. Universidad Autónoma de Ciudad Juárez.fany.solis@uacj.mx \\ *Autor de Correspondencia: Fany Thelma Solís-Rodríguez, correo electrónico: fany.solis@uacj.mx.
}

\section{RESUMEN}

El desarrollo de estrategias de mercadotecnia por parte de las empresas parecía ser estable, sin embargo, se ha visto afectado por la contingencia y el confinamiento debido a la pandemia por Covid-19. Con instrucciones sanitarias oficiales para impedir la propagación de la infección, se establecieron políticas de confinamiento que restringieron las actividades económicas a nivel nacional. Los microempresarios tuvieron que adaptar o modificar sus estrategias de mercadotecnia ante las restricciones, limitando la interacción directa con los consumidores y usuarios. La investigación tuvo como objetivo determinar las estrategias de mercadotecnia que implementaron las microempresas para la venta de productos y servicios en Ciudad Juárez, México ante la contingencia y el confinamiento por el Covid-19. El estudio fue tipo exploratorio y descriptivo, transversal, no experimental, con enfoque cualitativo basado en el paradigma interpretativo mediante el método de teoría fundamentada, empleando la técnica de entrevista. Algunas de las estrategias implementadas por los microempresarios fueron el uso del internet y las redes sociales en la publicidad, las barreras físicas y de señalación de distanciamiento, la reducción de la capacidad de personas dentro de los establecimientos, trabajos sobre pedido o encargos, la desinfección continua de las áreas de trabajo, entre otras.

Palabras clave: Estrategias de mercadotecnia; microempresarios; contingencia; covid-19.

Cómo citar:

Solís-Rodríguez, Fany Thelma. (2021). ESTRATEGIAS DE MERCADOTECNIA EN MICROEMPRESAS ANTE LA CONTINGENCIA POR COVID-19 EN CIUDAD JUÁREZ, MÉXICO: UN ESTUDIO CUALITATIVO. Revista de Investigaciones Universidad del Quindio, 33(S1), 19-31. https://doi.org/10.33975/riuq.vol33nS1.480 


\begin{abstract}
The development of marketing strategies by companies appeared to be stable, however, it has been affected by contingency and confinement due to the Covid-19 pandemic. With official health instructions to prevent the spread of infection, confinement policies were established that restricted economic activities at the national level. Micro-entrepreneurs had to adapt or modify their marketing strategies in the face of restrictions, limiting direct interaction with consumers and users. The research aimed to determine the marketing strategies implemented by micro-enterprises for the sale of products and services in Ciudad Juarez, Mexico in the face of the contingency and confinement by Covid-19. The study was exploratory and descriptive, transversal, non-experimental, with qualitative approach based on the interpretative paradigm using the method of informed theory, using the interview technique. Some of the strategies implemented by micro-entrepreneurs were the use of the internet and social networks in advertising, physical barriers and distance pointing, the reduction of the capacity of people within establishments, work on demand or commissions, the continuous disinfection of the work areas, among others.
\end{abstract}

Keywords: Marketing strategies, micro-entrepreneurs, contingency, covid-19.

\title{
INTRODUCCIÓN
}

En estos momentos, el mundo está atravesando por una etapa crítica en salud y economía, la cual ha sido propiciada por el virus Covid-19. La propagación del virus ha significado un patrón de cambio para las culturas de todo el mundo, pues ha obligado a las personas a modificar sus rutinas y hábitos cotidianos de cierta manera. Uno de ellos tiene que ver con la compraventa de productos, pues el mercado se ha detenido a causa del cierre de empresas, negocios, plazas y sucursales a nivel mundial, con el fin de proteger la integridad sanitaria de la población.

Ahora los microempresarios tienen como misión adaptarse al contexto que trae consigo el confinamiento, pues se ha limitado la interacción con los consumidores debido al aislamiento en sus hogares y cierre obligatorio de los negocios. Las estrategias de mercadotecnia han evolucionado, en parte por los desarrollos tecnológicos y las redes sociales. Sin embargo, la contingencia sanitaria por el Covid-19 ha obligado de manera coyuntural y hostil a que los microempresarios desarrollen o adapten nuevas estrategias para ofertar y vender sus productos y servicios, así como para adquirir bienes y servicios con sus proveedores.

En México, la contingencia sanitaria por Covid-19 comenzó en marzo de 2020, por lo que se obtuvo información al respecto de esa fecha por parte de microempresarios que residen en Ciudad Juárez, México. El estudio se orientó a las microempresas y las estrategias de mercadotecnia que se implementaron por razones de la contingencia, con el fin de determinar y comprender la manera de adaptarse al entorno socioeconómico que se vive. Lo anterior, ha orillado a los microempresarios a cambiar la modalidad original de venta de sus productos, ahora limitados al proporcionar un servicio en el establecimiento o convertir su servicio en algo distinto a lo que se ofrecía antes de la pandemia. Ante ello, tuvieron que realizar ajustes para que el negocio funcionara dentro de las medidas de prevención adecuadas impuestas por disposición gubernamental. 
La pregunta de investigación consistió en ¿qué estrategias de mercadotecnia han implementado los propietarios de microempresas ante el confinamiento por Covid-19? Se analizó la percepción de los microempresarios ante la necesidad de crear nuevas estrategias de mercadotecnia, así como sus principales dificultades y retos. Así, el objetivo de la investigación consistió en determinar las estrategias de mercadotecnia que implementaron los propietarios de las microempresas para la venta de productos y servicios en Ciudad Juárez, México ante la contingencia por Covid-19.

\section{MARCO TEÓRICO}

El diseño de estrategias mercadológicas es uno de los aspectos más importantes para las empresas, pues a través de estas se define la manera en que van a lograr las metas y objetivos de la empresa. A través de la literatura se han propuesto diversas estrategias en función del entorno, el tiempo y la situación individual y colectiva de la empresa, estas tienen un lapso de vida y requieren de una renovación, adaptación e innovación continua. Las estrategias mercadológicas tienen una relación intrínseca con el mercado, pues si éste cambia las estrategias también deben hacerlo, esto con el objetivo de satisfacer los deseos y necesidades de los consumidores. Sainz (2018) menciona que la mercadotecnia es clave para las ventas de una empresa, pues ayuda a identificar nuevos compradores y mercados, mejora la percepción del comprador con relación a la marca, y permite determinar y modificar el comportamiento de los compradores.

Es a través de la mercadotecnia que se puede llegar a conocer el mercado y por ende es importante poner énfasis en el diseño de estrategias de mercado. Selman (2017) propone una estrategia denominada reactivación, con la cual sugiere que tras una suspensión de actividades de la empresa es necesario reactivarla con un plan de trabajo, considerando la posibilidad de nuevos modelos de negocio y de gestión del servicio. Esta estrategia es sin duda un plan de acción que las empresas deberían concebir ante una crisis.

Según Ferrell y Hartline (2013), existen distintas formas de diseñar estrategias mercadológicas, pero la más común y efectiva es a través de un estudio de mercado, el cual consiste en una investigación profunda acerca de las distintas variables que intervienen en el mercado para comprender el comportamiento del consumidor y la relación que éste tiene con la adquisición de productos y servicios ofertados por las empresas. Sánchez (2020) señala que uno de los retos que enfrentan las empresas hoy en día es el desarrollo de estrategias que puedan aplicar para grupos masivos de consumidores, es decir, conforme pasa el tiempo los consumidores buscan satisfacer sus necesidades y deseos a través de productos personalizados y muy específicos, lo que limita la oportunidad de crear estrategias de mercado que abarquen a todos los segmentos de mercado que conforman sus clientes. Duviver (2020) afirma que el cliente racional se sitúa en una lógica de ventajas e inconvenientes, por lo que la oferta de la empresa debe demostrar su eficacia, mientras que el cliente debe sentirse seguro y convencido de que dicha oferta responde a su necesidad.

Las empresas deben tener en cuenta el constante cambio que sufren los mercados, solo el conocimiento del entorno les permitirá liderar ante una situación adversa. Al respecto, Mullins et al. (2007) coinciden en que los cambios en el ambiente del mercado hacen que los clientes reduzcan la importancia que dan a cierto atributo actual y los responsables de la mercadotecnia pueden tener dificultades para reposicionar una marca mediante la detección de un nuevo atributo percibido. Es decir, las características que se encuentran en tendencia y en la preferencia del consumidor pueden cambiar rápidamente a causa de factores externos a la empresa. Kotler et al. (2011) señalan que el entorno de 
mercadotecnia de una empresa lo forman todos aquellos agentes y fuerzas externas que afectan a su capacidad para desarrollar y mantener operaciones en beneficio de sus clientes objetivo, porque dicho entorno ofrece tanto oportunidades como amenazas y las empresas de éxito priorizan el observar y adaptarse al entorno cambiante.

Kotler y Armstrong (2012) explican que los métodos de venta están diseñados en función de las estrategias de mercado que las empresas utilizan para interactuar con sus consumidores a fin de comercializar, vender, distribuir y promocionar sus productos y servicios, de ahí que existan distintos métodos de venta, tales como la venta cara a cara, venta en línea y venta mixta. Según estos autores, la venta cara a cara es aquella que se realiza en la sucursal o establecimiento de la empresa y donde los consumidores acuden para poder adquirirlos; la venta en línea se lleva a cabo vía internet, la empresa oferta un catálogo de productos en línea, se realiza el pago vía electrónica y el producto es llevado hasta el domicilio del consumidor en una fecha previamente establecida, las desventajas de este método de venta comprenden el que los tiempos de entrega son inciertos, el índice de quejas de clientes es más alto y el número de devoluciones también; y la venta mixta, conformada por características de las dos estrategias anteriores, donde la empresa oferta los productos vía internet, pero la entrega de los productos se lleva a cabo en el establecimiento de la empresa.

Marulanda y Velázquez (2010) sostienen que el método que las empresas decidan utilizar tendrá un efecto en la aceptación y nivel de ventas, por lo que debe considerarse el nicho de mercado al que se dirige y definir si el sistema de venta es lo suficientemente accesible y eficiente para quienes dispondrán de él. A su vez, Porter (2015) plantea que existen diversas estrategias mercadológicas orientadas a la venta de productos y servicios, el autor las denomina ventajas competitivas, debido a la característica positiva que otorgan a la empresa a causa de su implementación, estas ventajas comprenden el liderazgo de costos, en la cual la empresa está capacitada para ofrecer en el mercado un producto a un precio inferior comparado a la oferta de las empresas oponentes; la diferenciación, donde las empresas le apuestan a aquellos mercados donde los compradores buscan características peculiares del producto distintas a las que ofrecen las empresas oponentes; y la del enfoque, que consiste en especializarse en un segmento del mercado y en ofrecer el mejor producto pensado expresamente para tal segmento.

\section{MÉTODO}

El estudio fue tipo exploratorio, descriptivo, transversal, con enfoque cualitativo basado en un paradigma interpretativo mediante el método de teoría fundamentada. Exploratorio porque dado un contexto de mercado atípico y coyuntural no se cuenta con un antecedente de confinamiento prolongado, salvo el H1N1 en 2008, sin embargo, las restricciones sanitarias no fueron radicales y el periodo de contagio en esa ocasión fue relativamente corto. Descriptivo porque se buscó determinar la percepción de los microempresarios sobre la situación de confinamiento y las circunstancias, estrategias y acciones que implementaron para la venta de sus productos y servicios.

En cuanto al paradigma interpretativo, este consiste en comprender a las personas y la percepción que tienen del mundo en el que viven y trabajan, por lo que desarrollan significados subjetivos a través de sus sentidos y experiencias con ciertos objetos o asuntos; dichos significados se forman a través de la interacción con otras personas, así como de las normas históricas y culturales que operan en la vida de los individuos, a partir de ello los investigadores generan o desarrollan inductivamente una teoría o patrón de significado (Creswell, 2014).

El método de teoría fundamentada permite observar el fenómeno en un intento de descubrir sus rasgos 
fundamentales y así explicar las regularidades constatadas (Boudon y Lazarsfeld, 1984). Este método otorga la posibilidad de encontrar conexiones entre temas y descubrir categorías en el contexto empírico observado (Dabenigno, 2017).

La técnica de recolección de información fue la entrevista estructurada, la cual se realizó vía remota mediante la plataforma Zoom durante el semestre agosto-diciembre de 2020 a microempresarios residentes en la frontera norte, específicamente en Ciudad Juárez, México. Ante las condiciones actuales y las limitantes tecnológicas de los sujetos de estudio la muestra teórica consistió en cinco participantes voluntarios, de quienes se obtuvo la saturación teórica en la quinta entrevista, dichos participantes son propietarios de los siguientes negocios:

1. Claudia. Taller de serigrafía

2. Andrés. Restaurante bar

3. José Luis. Jardín de eventos

4. Ricardo. Minisúper

5. Erick. Estudio fotográfico

Con respecto a la muestra teórica, Hernández et al. (2014) afirman que el valor que posee la investigación cualitativa se centra más en la profundidad de las descripciones y las categorías respecto al contexto que se estudia que en la generalización.

Una vez transcritas las entrevistas se procedió con la codificación cualitativa abierta o de primer plano para efectos de identificar segmentos, convertirlas en unidades de significado o categorías y asignarles códigos. Luego se siguió con la codificación axial o de segundo plano, para agrupar categorías en temas y conectarlas y con ello conformar la categoría central.

En cuanto al rigor en la investigación cualitativa, se consideró entrevistar a microempresarios cuyo contexto es el mismo, pero sus sectores económicos son distintos, esto con el fin de comparar y relacionar las experiencias durante el análisis y obtener mayor confiabilidad del estudio. Respecto a la credibilidad, Sullivan (2009) afirma que consiste en la capacidad de comunicar el lenguaje, así como los puntos de vista de los participantes; en tanto, Franklin y Ballau (2005) sostienen que la credibilidad es la adecuación referencial, es decir, la cercanía entre lo descrito y los hechos, por ello en este trabajo se presentan extractos de las narrativas de los microempresarios obtenidas a través de las entrevistas. En la cuestión de transferencia, la aplicación de los resultados a otros contextos, muestras o personas se considera que aún está en dicho proceso, ya que no se cuenta hasta el momento con estudios recientes al fenómeno de estudio.

\section{HALLAZGOS}

\section{Estrategias de venta antes de la contingencia}

Durante las entrevistas se cuestionó sobre las estrategias de venta, las cuales van desde la atención personalizada hasta la contratación de publicidad y estrategias de promoción de ventas.

a. Atención personalizada al cliente

Claudia: “...se atendía a las personas de manera normal en el establecimiento, los pedidos eran en 
persona y se dejaba un anticipo por el trabajo solicitado y cuando estaba listo las personas acudían a recoger el producto".

La estrategia venta de Claudia previa a la contingencia estaba centrada en el servicio al cliente, la cual realizaba de forma física en su establecimiento, teniendo trato personal con el cliente desde el inicio de la orden hasta la entrega del producto final en el mismo establecimiento. Según Kotler y Armstrong (2013), el servicio al cliente es un elemento de la estrategia del producto y funciona como servicio de apoyo en la venta.

b. Descuentos en el precio por venta o compra al mayoreo

Otra estrategia de venta era sobre la base del precio, pues al tener una estructura de costos establecida fue posible calcular el margen de ganancia y descuento adecuado sin alterar el precio competitivo ofrecido al público. De acuerdo con Kotler y Armstrong (2012), el precio es una estrategia que se utiliza en distintos términos según el ciclo de vida del producto, en el caso de madurez el precio se orienta a equiparar o en su caso abatir a los competidores.

Ricardo: "Podría decirse que comprar al mayoreo nos permite tener precios bajos y eso nos pone en la preferencia de nuestros clientes".

Erick: "Nuestro nicho principal son fotografías de bodas, quince años y fiestas, al ser eventos con grandes cantidades de personas podíamos revelar fotos en grandes volúmenes y así manejar bajos costos".

\section{c. Publicidad y promociones de venta}

Andrés: “...Invertir en publicidad para dar a conocer nuestros productos y que ahora estaban disponibles para llevar e implementamos el servicio a domicilio, así como algunas promociones temporales como $2 \mathrm{x} 1$ en hamburguesas y disminución ligera de precios para aumentar el número de visitas y posteriormente de ventas".

José Luis: “... Informando en las redes sociales de nuestro posible regreso y estar a la expectativa de cuando esto iniciará, ya una vez inicio (sic) tuvimos que promocionar nuestro nuevo servicio adaptado a la nueva necesidad".

En este caso, la contratación de publicidad y emplear promociones de venta permiten dar a conocer los nuevos productos y servicios que ofrece el establecimiento, más cuando la empresa es de reciente apertura y se desea posicionar la marca dentro de una localidad. A su vez, las promociones son un recurso para atraer clientes, retenerlos o incrementar su visita en días o temporadas clave, pues según Kotler y Armstrong (2013), la publicidad genera consciencia del producto e interés en el mercado, además enfatiza las diferencias, sus beneficios y mantiene la lealtad.

\section{Efectos de la contingencia y el confinamiento por Covid-19 en la empresa}

La entrevista permitió abordar el tema sobre los efectos que la contingencia y el confinamiento han tenido en las microempresas, con ello se obtuvo información de primera mano referente a las principales afectaciones al vender los productos o al ofrecer el servicio. 
Ricardo: "Como consecuencia de la pandemia tuvimos que dar un giro de cambio al negocio, tuvimos que imponer las medidas que las autoridades destinaron como esenciales: uso de cubrebocas, revisión de temperatura, sana distancia y restricción de un cliente por familia o grupo para evitar el conglomerado de personas".

Andrés: “... Pues en general fueron efectos negativos, nos vimos en la necesidad de dar convenios, modificar horarios y eso causó incertidumbre en nuestros empleados, ya que no se sabía cuándo terminaría esto, además de que las ganancias fueron bajísimas al grado que en ocasiones solo alcanzaba para cubrir gastos".

Lo anterior, requirió modificaciones en el recibimiento y atención al cliente, así como adaptaciones en la operación de la empresa por la necesidad de protección de los empleados, además de presentar una disminución de clientes, por ende, se afectó el nivel de la demanda; aun cuando en algunos casos se han mantenido las empresas en actividad, no ha sido con los niveles de venta anteriores a la contingencia.

Claudia: "El total de trabajos encargados por los clientes disminuyó en gran medida, nuestras ventas se redujeron en gran cantidad y los proyectos de compra de nueva maquinaria fueron pospuestos".

Erick: “... Disminución en la demanda de nuestros servicios, aunque también pudimos entender que no se estaba haciendo todo lo posible para generar ganancias”.

José Luis: “... Pues, el no operar de manera cotidiana durante la primera parte fue el principal, después el adaptar un servicio a la nueva manera de llevarlo a cabo”.

\section{Efectos de la contingencia en la cadena de proveedores}

Se cuestionó a los microempresarios sobre los proveedores y la cadena de suministro, con lo cual surgieron diferentes experiencias respecto a los retos para el abastecimiento.

Claudia: "Tuvimos que cambiar de proveedores, ya que los anteriores cerraron a causa de la pandemia, ya que traían su mercancía de Estados Unidos y el cierre de los puentes evita que transporten su mercancía aquí".

José Luis: "En base a los proveedores, como la mayoría de nuestros insumos, en específico los que tiene que ver con el mantenimiento y limpieza, realizamos un cambio en algunos productos por el cierre de la frontera, el cual no impacta directamente en la calidad del servicio, pero sí en el costo".

Ricardo: "Algunos proveedores informales querían elevar los precios de la mercancía, usando como pretexto la pandemia, por ejemplo, los proveedores de especias y de carne”.

Andrés: "El aumento de precios de algunos productos, y algunos productos que venían de El Paso (Texas, USA) tuvimos que sustituirlos con proveedores locales para tratar de otorgar la misma calidad y sabor que el cliente está acostumbrado".

Al considerar que Ciudad Juárez es una frontera y que algunos de los microempresarios tienen proveedores en la ciudad vecina de El Paso, Texas, USA, los efectos de la contingencia y el 
confinamiento modificó la relación con la cadena de proveedores, por lo cual los microempresarios tuvieron que buscar proveeduría que sustituyera los productos y servicios originales para su venta en la empresa, considerando además otros precios y calidad.

\section{Adaptaciones de la materia prima en el producto o servicio}

José Luis: “... Un poco, únicamente como ya mencioné el buscar los productos que adquirimos en proveedores locales".

Ricardo: “... Conseguimos proveedores de los nuevos productos que introducimos al negocio, además de conseguir más proveedores locales por la cuestión del cierre de las importaciones".

Erick: “... Como mencioné anteriormente; la materia prima comenzó a ser entregada a través de paquetería, representó un gasto extra, aunque me ahorró el tiempo de tener que ir al punto de recolección con mi proveedor".

Claudia: “... Un cambio de proveedor y además comenzamos a comprar otra variedad de materia prima, ya que comenzamos a vender artículos referentes a la pandemia como camisetas con frases del momento y cubrebocas con diseño".

Lorenzo: “... Pues el adquirir productos cuando el precio era más bajo de lo previsto en productos envasados y algunos cambios de proveedores por productos que venían del extranjero, obviamente en productos y en cuestión de ventas sin duda el servicio para llevar y de domicilio fue clave".

\section{Resultados de la adaptación de la materia prima}

Lorenzo: "Logramos mantener los costos de operación a un nivel al que estábamos acostumbrados y un nivel de ventas que nos permitiera aguantar este periodo de cambios".

José Luis: “... Positivos debido a que se logra mantener el servicio de la misma forma, aunque con un costo un poco más elevado".

Ricardo: “... Pudimos atender la demanda de nuestros consumidores respecto a los productos de necesidad actual, los requeridos para mantenerse seguro de la pandemia”.

Erick: “... Mi materia prima llegaba con pequeños retrasos debido a la logística de la paquetería, así que tuve que pedir con más días de anticipación”.

Claudia: "Logramos estabilizar un poco las ventas en consideración a la baja demanda que teníamos al iniciar la pandemia, además de que logramos destacar con los nuevos productos que incorporamos al portafolio de trabajos".

\section{Estrategias de mercadotecnia ante la contingencia y el confinamiento por el Covid-19}

José Luis: "El ofrecer un ambiente seguro, donde las personas puedan llevar un convivio de forma segura, entonces el ofrecer este ambiente dentro de nuestro lugar donde se toman todas las medidas de prevención por parte de nuestros empleados y donde se sienta cómodo el cliente es la principal 
y claro darlo a conocer por parte de nuestras redes sociales".

Andrés: "Implementar el servicio a domicilio con un costo bajo el cual nos permitiera tanto al cliente como nosotros obtener beneficios de él, también ofrecimos dos nuevos productos e invertir en la publicidad de redes sociales y generar todos los días nuevo contenido sobre promociones fue clave, desde el inicio del confinamiento hasta hoy en la nueva normalidad donde ya se permite público en general".

Claudia: "Una estrategia fue reducir el aforo de gente, de esta manera asegurábamos que al día siguiente tuviéramos trabajo, además comenzamos a regalar pequeños artículos que no representaran grandes costos para que así la gente pudiera apreciar nuestro trabajo, comenzamos a recibir pedidos a través de páginas en redes sociales y pagos a través de depósitos bancarios, lo cual permitió que la gente comprara desde la comodidad de su hogar".

Parte de las estrategias de mercadotecnia se enfocaron en ofrecer un establecimiento seguro para los clientes reforzando la prevención en los empleados y un ambiente propicio para la venta de los productos y captación de clientes. Otra estrategia importante fue ofrecer el servicio o entrega a domicilio, reduciendo al mínimo la exposición al contagio, no obstante, trajo consigo cambios en la variedad y presentación de productos, así como en las promociones considerando el uso primordial de las redes sociales para generar expectativas de calidad en el público. Además, se recurrió al uso de la atención telefónica y ventas con terminal electrónica.

Ricardo: “... Comenzamos a recibir pedidos a través de la aplicación de WhatsApp, así la gente solo pasaba a recibir y pagar su pedido y evitaba entrar al establecimiento, también comenzamos a vender artículos de temporada como cubrebocas, gel antibacterial y desinfectantes especiales".

Erick: "Mi principal estrategia fue rebajar precios y ampliar los paquetes de fotografías, además de comenzar a manejar la fotografía de producto. Además, comencé a destacar la desinfección dentro de nuestro negocio al momento de promocionar nuestros servicios".

Con base en lo anterior, las estrategias de mercadotecnia se enfocaron en ofrecer una atención al cliente de manera segura, la variedad de productos, difusión de sus servicios durante la contingencia y acondicionar un ambiente propicio para garantizar la seguridad en los clientes durante la visita a su establecimiento.

\section{Beneficios esperados con las estrategias de mercadotecnia implementadas}

Claudia: "Que la gente consumiera de igual manera a que si no estuviera la pandemia y que pudiera sentirse segura en nuestras instalaciones".

Andrés: "Que el público pudiera tener nuestros platillos con seguridad, higiene y el mismo sabor sin necesidad de salir de su hogar o exponerse a algún posible contagio y nos permitiera amortizar el golpe de cambios que originó el confinamiento".

Las anteriores respuestas se orientan al cliente, en específico que éste se sintiera con la seguridad de acudir al establecimiento y que las circunstancias de pandemia no modificaran la manera ni la experiencia de adquirir sus productos. 
José Luis: "El recuperar un poco del margen perdido ya por un gran tramo de la temporada de eventos para cubrir nuestros gastos operativos".

Erick: "En primera instancia era incrementar las ventas, al pasar los meses y ver que la situación no mejoraba, solo esperábamos no tener que cerrar, ya que se tenían que seguir cubriendo gastos fijos como el arrendamiento del local y servicios básicos".

Ricardo: "Conservar las ventas promedio del negocio y mejorar el servicio al cliente en cuando al cuidado de la salud".

La mayoría de los entrevistados optaron por adquirir productos con proveedores locales, así como ofrecer nuevos productos o realizar nuevas adaptaciones con servicio a distancia, con el objetivo principalmente de mantener costos de operación, entender la demanda de los clientes con la misma calidad y precio, así como lograr un nivel de ventas óptimo que permitiera a la empresa mantener su actividad.

\section{Retos de las microempresas ante la contingencia y el confinamiento}

Se cuestionó a los entrevistados sobre los efectos de la contingencia y el confinamiento, a su vez se realizó un extracto de la percepción que los microempresarios tienen ante lo que consideran los retos que han tenido que enfrentar para continuar con su actividad en el mercado, los extractos textuales se incluyen en la Tabla 1.

Tabla 1. Retos en las microempresas ante la contingencia y el confinamiento

\begin{tabular}{|l|l|}
\hline \multicolumn{1}{|c|}{ Entrevistado } & \multicolumn{1}{c|}{ Retos de las microempresas } \\
\hline $\begin{array}{l}\text { Claudia. Taller } \\
\text { de serigrafía }\end{array}$ & $\begin{array}{l}\text { "Reducción de las ventas en gran medida a principios de la cuarentena y gastos } \\
\text { extra en las medidas implementadas, como el gel antibacterial para los clientes, } \\
\text { etc." }\end{array}$ \\
\hline $\begin{array}{l}\text { Andrés. } \\
\text { Restaurante bar }\end{array}$ & $\begin{array}{l}\text { "Señalar espacios de sana distancia, proporcionar gel antibacterial, tomar } \\
\text { temperatura, uso de cubrebocas, lavado de manos constantemente, desinfectar } \\
\text { cada 15 minutos el espacio laboral". }\end{array}$ \\
\hline $\begin{array}{l}\text { José Luis. Jardín } \\
\text { de eventos }\end{array}$ & $\begin{array}{l}\text { "Adaptarse sin duda a las nuevas normas de higiene impuestas por instituciones } \\
\text { gubernamentales, el desinfectar áreas comunes. El aforo máximo lo ajustamos } \\
\text { para mantener sana distancia". }\end{array}$ \\
\hline $\begin{array}{l}\text { Ricardo. } \\
\text { Minisúper }\end{array}$ & $\begin{array}{l}\text { "Limitaciones para ofrecer los servicios de la manera habitual, además una } \\
\text { disminución significativa de la demanda". }\end{array}$ \\
\hline $\begin{array}{l}\text { Erick. Estudio } \\
\text { fotográfico }\end{array}$ & $\begin{array}{l}\text { "Se perdieron clientes y se destinó parte de las ganancias a las nuevas estrategias } \\
\text { a la infección y se tuvo que remplazar sus puestos temporalmente, lo que generó } \\
\text { un costo extra". }\end{array}$ \\
\hline
\end{tabular}

Nota. Se presenta un extracto de la narrativa de los entrevistados sobre lo que consideran los principales retos en las microempresas ante la contingencia y el confinamiento. Elaboración propia con base en la información obtenida de las entrevistas.

Una vez sistematizada la codificación abierta de las categorías referentes a la propia lectura de las 
Se identificaron acciones y retos que han presentado los propietarios de microempresas ante el confinamiento, entre ellas: el retraso en el recibimiento de las materias primas por el cierre de fronteras, el límite de atención a clientes por las restricciones de salud y la disminución del volumen de ventas por la actual recesión económica.

En virtud del análisis es posible identificar acciones que constituyen estrategias de implementación por parte de las microempresas en un escenario próximo:

1. Previsión en los pedidos de materias primas: Guardar un inventario básico de materia prima que permita continuar con la producción en caso de un inconveniente con la entrega por parte del proveedor.

2. Inversión en publicidad y canales de difusión alternos para la promoción: Incursionar en nuevos medios de información para promocionar los productos o servicios que se ofrecen.

3. Comunicación de las medidas preventivas de salud implementadas en la empresa: Dar a conocer a los clientes y consumidores las medidas y restricciones sanitarias en las instalaciones para hacerlos sentir seguros al acudir a comprar o consumir.

4. Adaptación del modelo de negocio: Integrar productos y servicios acordes a la situación, adaptar los procesos para que estos no se vean afectados, implementar estrategias redituables y capaces de hacer frente al contexto.

Esta investigación permitió tener una mejor comprensión de la situación que atraviesan las microempresas de la localidad ante el fenómeno de salud y la afectación en los diferentes aspectos que involucran las actividades de las empresas, así como las acciones y soluciones que han implementado.

Por último, el tema de investigación sigue siendo importante, pues la situación de crisis de salud y económica aún se encuentra presente, es difícil definir estrategias de mercadotecnia futuras, pues existe incertidumbre en el mercado. Por ello, es seguro que será un tema de referencia para futuras investigaciones y del que puede obtenerse información empresarial relevante.

\section{REFERENCIAS}

1. Boudon, R., \& Lazarsfeld, P. (1984). Metodología en las ciencias sociales. Conceptos e índices. Laia. http://anchecata.colmich.edu.mx/janium/Tablas/tabla53132.pdf

2. Creswell, J. W. (2014). Research Design quantitative, qualitative, and mixed methods approaches. Sage.

3. Dabenigno, V. (2017). La sistematización de datos cualitativos desde una perspectiva procesual. De la transcripción y los memos a las rondad de codificación y procesamiento de entrevistas. En P. Borda, V. Dabenigno, B. Freidin, \& M. Guelman (Eds.), Estrategias para el análisis de datos cualitativos (pp. 22-71). Centro de Documentación e Información, IIGG. https://ri.conicet. gov.ar/bitstream/handle/11336/112116/CONICET_Digital_Nro.2d904b6c-1ee4-493f-954086f04528fba2_A.pdf?sequence $=2$

4. Duviver, J. (2020, diciembre 15). Aprende a realizar un buen estudio de mercado. 50minutos.es. https://www.50minutos.es/libro/aprende-a-realizar-un-buen-estudio-de-mercado/

5. Ferrell, O., \& Hartline, M. (2013). Estrategia del Marketing. Cengage Learning. http://www. elmayorportaldegerencia.com/Libros/Mercadeo/\%5BPD\%5D\%20Libros\%20-\%20Estrategia\%20 de\%20Marketing.pdf

6. Franklin, C., \& Ballau, M. (2005). Reliability and validity in qualitative research. En R. Grinnell, \& Y. Unrau (Eds.), Social work: Research and evaluation. Quantitative and qualitative approaches (pp. 438-449). Oxford University Press. https://scholarworks.wmich.edu/books/306/

7. Hernández, R., Fernández, C., \& Baptista, P. (2014). Metodología de la Investigación. Mc Graw 
Hill.

8. Kotler, P. \& Armstrong, G., (2012), Marketing. Pearson.

9. Kotler, P. \& Armstrong, G. (2013). Fundamentos de marketing. Pearson Educación.

10. Kotler, P., Bowen, J., Mackens, J., García, J., \& Flores, J. (2011). Marketing Turístico. Pearson.

11. Marulanda, I., \& Velázquez, A. (2010). Formulación de un plan estratégico de marketing para la empresa freskaromas. [Disertación de tesis no publicada]. Universidad Tecnológica de Pereira. http://repositorio.utp.edu.co/dspace/bitstream/handle/11059/1739/6588M389.pdf?sequence=1

12. Mullins, J., Walker, O., Boyd, H., \& Larrenche, J. (2007). Administración del Marketing, un enfoque en la toma estratégica de decisiones. Mc Graw Hill.

13. Porter, M. (2015). Ventaja Competitiva: crear y sostener un desempeño superior. Patria.

14. Sainz J. (2018) El plan de marketing digital en la práctica. ESIC.

15. Sánchez, G. (2020) Nuevas estrategias de marketing en las PYMES de Córdoba. Estudio de casos. [Disertación de tesis no publicada]. Universidad de Córdoba. https://repositorio. unicordoba.edu.co/bitstream/handle/ucordoba/2996/Garcia\%20Sanchez\%20Gustavo\%20A. pdf? sequence $=1 \&$ isAllowed $=\mathrm{y}$

16. Selman, H. (2017). Marketing Digital. Ibukku.

17. Sullivan, L. E. (2009). The SAGE Glossary of the Social and Behavioral Sciences. SAGE. 